Schweizerisches Jahrbuch für Entwicklungspolitik

27-2 | 2008

Migration und Entwicklung : Eine Zweckallianz

\title{
Blickpunkt: Informelle Rücküberweisungen der Afghanen : Strategische Streuung
}

\section{Alessandro Monsutti}

\section{(2) OpenEdition}

12 Journals

Electronic version

URL: http://journals.openedition.org/sjep/335

DOI: $10.4000 /$ sjep.335

ISSN: 1663-9677

Publisher

Institut de hautes études internationales et du développement

Printed version

Date of publication: 1 décembre 2008

Number of pages: 163-167

ISBN: 978-2-940415-08-3

ISSN: 1660-5926

\section{Electronic reference}

Alessandro Monsutti, « Blickpunkt: Informelle Rücküberweisungen der Afghanen : Strategische Streuung ", Schweizerisches Jahrbuch für Entwicklungspolitik [Online], 27-2 | 2008, Online erschienen am: 31 Mai 2010, abgerufen am 07 September 2020. URL : http://journals.openedition.org/sjep/335 ; DOI : https://doi.org/10.4000/sjep.335 


\title{
Blickpunkt
}

\section{Informelle Rücküberweisungen der Afghanen: Strategische Streuung}

\author{
Alessandro Monsutti*
}

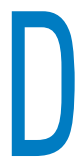

er Konflikt in Afghanistan nach dem kommunistischen Staatsstreich von 1978 und der sowjetischen Invasion von 1979 verursachte eine der massivsten Bevölkerungsbewegungen des späten 20. Jahrhunderts. Anfang der 90er-Jahre zählte das Flüchtlingshochkommissariat der Vereinten Nationen (UNHCR) über sechs Millionen afghanische Flüchtlinge, die sich grösstenteils auf Pakistan und den Iran verteilten'. Damals bildeten die Afghanen weltweit die grösste Gruppe Vertriebener; etwa die Hälfte dieser Personen fielen unter das Mandat des UNHCR. Die humanitären Organisationen und die Medien tendierten dazu, diesen Exodus als traumatisch zu beschreiben, was jedoch nicht immer der Fall war. In einer langfristigen historischen Perspektive muss der ausserordentliche Charakter des Exodus im Übrigen relativiert werden². Afghanen sind Nomaden, die ihre Herden im Verlauf der Jahreszeiten zum besten Weideland führen und die Wanderschaft nutzen, um mit der sesshaften Bevölkerung Handel zu treiben; Afghanen sind Bergbewohner, die in der Stadt oder im Flachland einen Saisonjob suchen, Pilger, Deserteure, Eroberer kurz, Afghanen besitzen langjährige Erfahrungen mit den verschiedensten Spielarten der Migration.
Heute wie gestern überweisen die Afghanen - sowie auch Migranten- und Flüchtlingsgruppen anderer Herkunft - grosse Geldsummen an ihre Familien, die im Heimatland geblieben sind. Die Untersuchung dieser Heimatüberweisungen ist in vielerlei Hinsicht aufschlussreich. Erstens zeigt sich, dass die Flucht vor Gewalt mit einer eigentlichen Migrationsstrategie nicht immer unvereinbar ist. Zweitens haben diese Geldsendungen bedeutende soziale und ökonomische Konsequenzen für die Herkunftsregionen der Flüchtlinge. Drittens und schliesslich wirken sie als Anreiz und Orientierung für die künftigen Migrationsbewegungen: Die Flüchtlinge informieren die zu Hause gebliebenen Familienmitglieder über die Möglichkeiten an unterschiedlichen Aufnahmeorten. Von Migranten oder von Flüchtlingen vorgenommene Geldsendungen zeigen das Vorhandensein sozialer Netze auf, die entfernte Orte miteinander verbinden. Im Fall Afghanistans ist die Diaspora nicht nur als leidvolle Auswirkung von Krieg und Armut zu sehen, sondern auch als Ergebnis eines bewussten Plans: Die Mitglieder der Familienclans versuchen, dadurch ihre Überlebensmittel zu diversifizieren und die Risiken der Unsicherheit, denen sie in ihrem Herkunftsland (anhaltende Kämpfe) und im Aufnahmeland

* Lehrbeauftragter am IHEID.

Dazu müssten noch rund anderthalb Millionen intern vertriebene Personen gezählt werden. R. Colville, „Afghan Refugees: Is International Support Draining Away after Two Decades in Exile?", Refuge, Bd. 17. Nr. 4, 1998, S. 6-11.

2 M.J. Hanifi, „Anthropology and the Representations of Recent Migrations from Afghanistan“, in E.M. Gozdziak and D.J. Shandy (eds.), Rethinking Refuge and Displacement: Selected Papers on Refugees and Immigrants, Bd. 8. Arlington, American Anthropological Association, 2000, S. 291-321. 
(Polizeikontrollen, Ausweisungswellen) ausgesetzt sind, zu verringern.

Ein erneuter Blick auf die Migrationsströme zeigt, dass sie häufig weder definitiv noch provisorisch sind. Daher wäre es zutreffender, über sich wiederholende Reisen in mehrere Richtungen zu sprechen. IIm Fall Afghanistan fällt dies besonders auf. Zum einen sind nur sehr wenige afghanische Flüchtlinge nach der ersten Ausreise nie mehr in ihr Land zurückgekehrt; zum anderen zählen die allermeisten Familiengemeinschaften mindestens ein Familienmitglied im Ausland. Auf Migration und Exil folgen also nicht unweigerlich die Integration im Empfangsland oder die endgültige Rückkehr in das Herkunftsland; die Migrationsbewegungen setzen sich kontinuierlich fort, sodass letztlich eine eigentliche transnationale Gemeinschaft entsteht. Die Afghanen haben ein „Umlaufgebiet" eingerichtet, um eine Formulierung des Soziologen Alain Tarrius zu verwenden ${ }^{3}$.

Beispielhaft dafür ist die Volksgruppe der Hazara. Die Hazara aus dem unfruchtbaren Berggebiet im Herzen Afghanistans sind mehrheitlich Schiiten und werden von der sunnitischen Bevölkerungsmehrheit mit Misstrauen und Herablassung behandelt. Armut und politische Diskriminierung trieben in der Vergangenheit zahlreiche Hazara aus ihrer Herkunftsregionen auf die Suche nach einem Arbeitsplatz in die Städte Afghanistans oder in die Nachbarländer Iran und Pakistan. Einige zogen auch in die Vereinigten Arabischen Emirate, nach Kuwait oder SaudiArabien. Während des Kriegs gewannen diese seit Langem bekannten Migrationsbewegungen ein beispielloses Ausmass. Wie andere Segmente der afghanischen Bevölkerung haben die Hazara äusserst effiziente Migrationsnetze geknüpft, die auf den zwischen Iran, Pakistan und Afghanistan verteil- ten Mitgliedern der Familienclans beruhen. Immer mehr Hazara liessen sich in Europa, Nordamerika, Australien und Neuseeland nieder. Diese umfassende Gruppe bildet ein eigentliches transnationales System; Strategien, die an einem bestimmten Ort entwickelt werden, können nur im Zusammenhang mit den anderswo geltenden Strategien verstanden werden. Jeder Wohnort weist Vorund Nachteile auf. In Afghanistan erlebten die Hazara zwar einen politischen Emanzipationsprozess, aber die allgemeine Lage des Landes - Sicherheit, Wirtschaft - bleibt weiterhin düster. In Pakistan liess sich die überwiegende Mehrheit der Hazara in der Hauptstadt von Belutschistan, Quetta, nieder. Quetta gewährte ihnen Zuflucht, weil sich bereits im späten 19. Jahrhundert, als die Stadt zu Britisch-Indien gehörte, eine Hazara-Gruppe dort niedergelassen hatte. Quetta bot den Migranten und Flüchtlingen ein Leben in relativer Freiheit, allerdings mit dürftigen ökonomischen Chancen. Im Iran dagegen fällt es zwar ziemlich leicht, in der Baubranche, in einer Getreidemühle oder als Landwirtschaftsarbeiter ein bescheidenes Auskommen zu finden, aber die Familien können sich wegen des Drucks durch die Behörden der Islamischen Republik und wegen der regelmässigen Ausweisungswellen kaum dauerhaft niederlassen.

Ein Hazara-Migrant, der in den Iran gezogen ist und seine bescheidenen Ersparnisse seiner Familie in Afghanistan schickt, kann dazu nicht das formelle Bankensystem benutzen. Erstens hat er häufig keine offiziellen Ausweispapiere. Zweitens gab es in den 80erund 90er-Jahren in Hazarajat praktisch kein Bankennetz; seit dem Fall der Taliban im Jahr 2001 hat es sich in Afghanistan schrittweise ausgedehnt. Deshalb vertraute der Migrant seine Scherflein einem auf informelle Geldsendungen spezialisierten Geschäftsmann an, lokal als hawaladar bekannt (von hawala

3 A. Tarrius, "Territoires circulatoires des entrepreneurs commerciaux maghrébins de Marseille: du commerce communautaire aux réseaux de l'économie souterraine mondiale", Journal des anthropologues, Nr. 59, 1995, S. 15-35. 
(Geld), "Transfer", auch „Kreditbrief" oder „Scheck”). Der Migrant kontaktiert nach Möglichkeit einen bekannten hawaladar, d.h. entweder ein Mitglied der Grossfamilie oder jemanden, der aus dem gleichen Tal oder Bezirk kommt. Bei entfernterer Bekanntschaft wird ein Vermittler eingeschaltet, der für die beiden Partner als Bürge auftritt. Der hawala- dar übergibt den Geschäftspartnern ein Schreiben, das die Einzelheiten der Transaktion festhält (Brief b im Schema unten); der Migrant erhält ein anderes Schreiben (Brief a), das er über einen Freund, der auf Heimatbesuch geht, an seine Familie in Afghanistan schickt.

\section{Schema: Geldüberweisungen zwischen Iran, Pakistan und Afghanistan in den 90er-Jahren}

IRAN

PAKISTAN

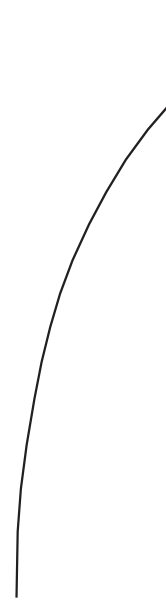

Brief a

AFGHANISTAN

Brief a
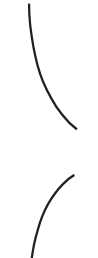

Brief b

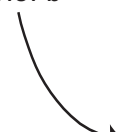

Arbeiter

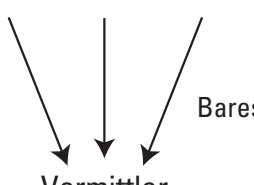

Vermittler

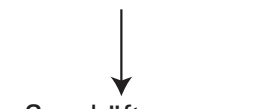

Geschäftsmann

Banküberweisung oder Schmuggel
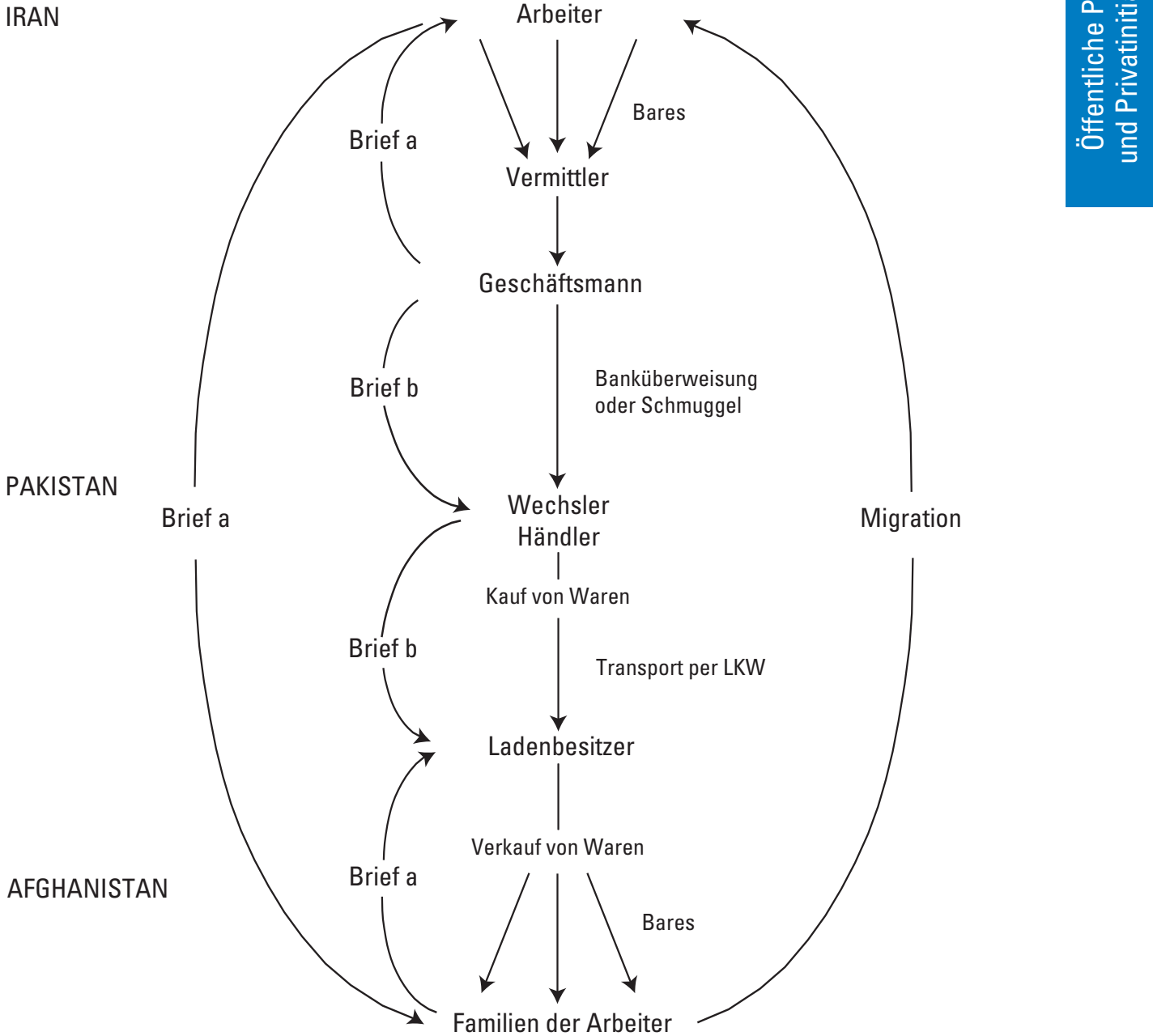

Quelle: A. Monsutti, Guerres et migrations: réseaux sociaux et stratégies économiques des Hazaras d'Afghanistan, Neuchâtel, Institut d'ethnologie; Paris, Maison des sciences de l'homme, 2004, S. 237. 
Nach dem Fall des Taliban-Regimes und der Einsetzung einer von der internationalen Staatengemeinschaft unterstützten Regierung in Kabul Ende 2001 wurden die meisten Geldüberweisungen nach Afghanistan direkt aus dem Iran abgewickelt. In den 90erJahren dagegen war es wegen der Spannungen zwischen dem grossen schiitischen Land und den sunnitischen Fundamentalisten sehr riskant, die Grenze zwischen den beiden Ländern zu überschreiten. Der hawaladar nahm das Geld im Iran entgegen und sandte es nach Pakistan - entweder als Banküberweisung oder in Naturalform (besonders Fertiggüter wie Kunststoffartikel, Bekleidung oder Stoffe). In Pakistan nahm ein Partner die Geldbeträge oder Produkte in Empfang und verkaufte sie vor Ort mit Gewinn weiter. Mit diesen Beträgen konnte er weitere Profite erzielen (z.B. auf dem Geldwechselmarkt), oder aber er benutzte sie für den Kauf anderer Waren (Mehl, Reis, Speiseöl, Tee, Zucker, Dampfkochtöpfe, Plastiksandalen usw.). Diese Waren wurden per Lastwagen bis nach Hazarajat befördert, wo ein dritter Partner einen Laden betrieb. Der Ladeninhaber zahlte mit dem Verkaufserlös die Familien der Migranten aus. Auf dieses Geschäft wurde nur eine geringe Gebühr erhoben ${ }^{4}$, weil der hawaladar die Gewinne im Wesentlichen daraus bezog, dass er mit wenig Eigenkapital Geschäfte machen konnte. Trotz der Traumata von Krieg und Exil waren die Hazara so in der Lage, die geografische Diaspora und die daraus hervorgehende wirtschaftliche Diversifizierung zu nutzen und sehr effiziente transnationale Kooperationsstrukturen aufzubauen.

Wie der vorliegende Band des Jahrbuchs zeigt, lösen die Auswirkungen der Geldsendungen in die Herkunftsregionen der Migranten lebhafte Diskussionen aus. Einerseits kritisieren bestimmte Autoren, dass dieses Geld häufig für den Kauf von Konsumwaren und Prestigeartikeln dient oder verprasst wird und so die sozialen Ungleichheiten verschärft. Andererseits betonen immer mehr Entwicklungsorganisationen dagegen die potenziell positive Rolle solcher Geldsendungen, weil sie den lokalen Wirtschaften Kapital zur Verfügung stellen, das anschliessend produktiv investiert werden kann. Im Fall Afghanistans wäre es sicherlich von Vorteil, wenn das hawala-System durch ein Bankensystem ergänzt würde: Damit kann das Kapital in Kredit- und Investitionsfonds umgewandelt werden und so zur Instandsetzung der Infrastrukturen des Landes dienen und zur Schaffung von Unternehmen beitragen. Obwohl die Emigration junger Männer langfristig mit Blick auf den Wiederaufbau Afghanistans problematisch scheint, hat sich diese Überlebensstrategie bewährt. Dank dem hawala-System hatten zahlreiche afghanische Familien genug zu essen, während sich die humanitäre Arbeit während der sowjetischen Besatzung vor allem auf die Flüchtlinge konzentrierte und in den 90erJahren zum Erliegen kam, bevor sie nach dem Fall der Taliban 2001, wenn auch in sehr unstrukturierter Form, wieder neue Bedeutung gewann.

Neben dem eigentlichen ökonomischen Beitrag bzw. den Grenzen ist vor allem die soziale Dimension der Geldüberweisungen durch Migranten von Belang. Auf diesem Weg bleiben die in alle Winde zerstreuten Menschen in Kontakt und bilden weiterhin eine Hilfs- und Solidaritätsgemeinschaft. Dieser Aspekt ist wesentlich: Heimatüberweisungen tragen dazu bei, trotz der Zerstreuung und im vorliegenden Fall trotz Krieg soziale Beziehungen zu schaffen und zu erneuern. Darin ist eine zentrale Rolle der Geldsendungen zu sehen. Das hawalaSystem - ein komplexes Beziehungsgeflecht von Kooperation und Konkurrenz, Vertrauen und Misstrauen - zeigt, dass die geografische Verzettelung und die sich daraus ergebende ökonomische Diversifizierung zur Risi-

In der Regel höchstens 3\% zwischen Iran, Pakistan und Afghanistan, häufig mehr auf Sendungen aus einem westlichen Land. 
koverteilung beitragen und sich zu einer dass die afghanische Gesellschaft trotz den geplanten Strategie entwickeln können. In Kämpfen und der Vertreibung von mehreren diesem Sinne bildet das hawala-System mit Millionen Menschen nicht im Hobbes'schen einen Erklärungsfaktor für die Tatsache, Chaos versunken ist.

\section{Zitierte Referenzen}

Colville, Rupert, „Afghan Refugees: Is International Support Draining Away after Two Decades in Exile?”, Refuge, Bd. 17, Nr. 4, 1998, S. 6-11.

Hanifi, M. Jamil, „Anthropology and the Representations of Recent Migrations from Afghanistan“, in E.M. Gozdziak and D.J. Shandy (eds.), Rethinking Refuge and Displacement: Selected Papers on Refugees and Immigrants, Bd. 8, Arlington, American Anthropological Association, 2000, S. 291-321.

Monsutti, Alessandro, Guerres et migrations: réseaux sociaux et stratégies économiques des Hazaras d'Afghanistan, Neuchâtel, Institut d'ethnologie; Paris, Maison des sciences de I'homme, 2004.

Tarrius, Alain, „Territoires circulatoires des entrepreneurs commerciaux maghrébins de Marseille: du commerce communautaire aux réseaux de l'économie souterraine mondiale“", Journal des anthropologues, Nr. 59, 1995, S. 15-35. 